\title{
A Chemical Reporter Strategy to Probe Glycoprotein Fucosylation
}

\author{
David Rabuka ${ }^{1}$, Sarah C. Hubbard ${ }^{1}$, Scott T. Laughlin ${ }^{1}$, Sulabha P. Argade ${ }^{5}$, and Carolyn R. \\ Bertozzi $^{1,2,3,4}$ \\ ${ }^{1}$ Department of Chemistry, University of California, Berkeley, CA \\ ${ }^{2}$ Department of Molecular and Cell Biology, University of California, Berkeley, CA \\ ${ }^{3}$ Howard Hughes Medical Institute, University of California, Berkeley, CA \\ ${ }^{4}$ Molecular Foundry, Lawrence Berkeley National Laboratory, Berkeley, CA \\ ${ }^{5}$ Glycotechnology Core Resource, University of California, San Diego, La Jolla, CA
}

\begin{abstract}

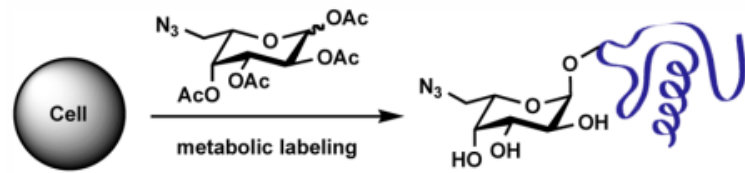

Glycoprotein

Fucosylated glycoproteins are involved in many cell-cell recognition events and are markers of embryonic and malignant tissue. Here we report a method for rapid profiling of fucosylated glycoproteins from human cells using 6-azido fucose as a metabolic label.
\end{abstract}

It has been estimated that $50 \%$ of the proteins from eukaryotic cells are posttranslationally modified by glycosylation. ${ }^{1}$ However, for most glycoproteins, the types of glycans attached to the polypeptide have not been defined, nor are their functions understood. Thus, the field of glycomics has focused considerable attention on the development of methods for profiling protein glycosylation at the systems level. ${ }^{1}$ Our contribution to this effort is the chemical reporter strategy for marking protein-associated glycans with bioorthogonal functional groups such as the azide. ${ }^{2}$ Metabolic labeling of specific glycan subtypes with azido sugar precursors enables their subsequent covalent reaction with fluorescent probes for visualization, or with affinity tags for enrichment and proteomic analysis. Several azidespecific chemistries can be employed in these experiments, including the Staudinger ligation, $\mathrm{Cu}(\mathrm{I})$-catalyzed azide-alkyne condensation (i.e., "click chemistry"), or strainpromoted [3+2] azide-alkyne cycloaddition. ${ }^{2}$ Mucin-type O-linked glycoproteins, ${ }^{3}$ sialylated glycoproteins ${ }^{4}$ and cytosolic O-GlcNAc-modified proteins ${ }^{5}$ are examples of glycoprotein classes that have been studied using the chemical reporter strategy. Here we report the extension of the method to fucosylated glycoproteins.

Among the nine monosaccharide building blocks employed in vertebrates, fucose is distinguished by its contribution to numerous epitopes associated with cell-cell interactions

Correspondence to: Carolyn R. Bertozzi.

Supporting Information Available: Synthetic procedures, spectral data for all compounds, experimental procedures for Western blots, flow cytometry and HPAEC-PAD. This material is available free of charge via the Internet at http://pubs.acs.org. 
and regulation of protein function. For example, fucose is a component of the tetrasaccharide sialyl Lewis ${ }^{\mathrm{x}}$, a determinant of the selectin ligands that mediate leukocyteendothelial interactions at sites of inflammation. ${ }^{6}$ Overexpression of sialyl Lewis ${ }^{\mathrm{x}}$ is characteristic of many cancers ${ }^{7}$ and glycoproteins displaying Lewis ${ }^{\mathrm{x}}$ epitopes promote embryonic cell adhesion and have important roles in neurogenesis. ${ }^{8}$ Fucose is also a terminal sugar on a number of tumor-associated antigens, including the tetrasaccharide Lewis $^{\mathrm{y}}$, a modification of carcinoembryonic antigen (CEA) in malignant colon cancer. ${ }^{9}$ The multitude of fucosylated antigens found upregulated in cancerous tissue provides an opportunity to exploit these glycans as potential cancer biomarkers. ${ }^{10}$ Fucose is also found directly attached to proteins via serine or threonine residues in a rare form of protein glycosylation known as O-linked fucosylation. This modification is found on epidermal growth factor (EGF) repeats in the developmental switch Notch. ${ }^{11}$ The ability to profile fucosylated glycoproteins from complex cell or tissue samples might therefore reveal new cancer biomarkers and provide a means to study changes in fucosylation associated with development.

Our approach exploits the fucose salvage pathway for the incorporation of azido analogs into fucosylated glycans. This pathway begins with the uptake of free fucose, followed by phosphorylation by fucose kinase and conversion to GDP-fucose by GDP-fucose pyrophosphorylase (Fig. 1). ${ }^{8}$ Fucosyltransferases, 13 of which have been identified in humans, transfer the fucose residue to glycans or proteins within the secretory pathway and the modified glycoproteins are delivered to the cell surface or secreted. Although the earlier enzymes have not been studied in detail with respect to unnatural substrate tolerance, at least two fucosyltransferases have demonstrated in vitro activity on GDP-fucose analogs bearing C6-modifications on the pyranose ring. ${ }^{12}$

We synthesized three azido fucose analogs (compounds 1-3, Fig. 2) and probed for their incorporation into glycoproteins in the human T lymphoma cell line Jurkat. Compounds $\mathbf{1}$ and $\mathbf{3}$ were prepared as previously reported in the literature ${ }^{13,12}$ and compound $\mathbf{2}$ was synthesized as shown in Scheme 1. Briefly, thiophenyl glycoside $\mathbf{5}^{14}$ was protected as the butane 2,3-diacetal (6) and the 4-OH was subjected to a double displacement process to introduce the azide (9). Global deprotection and acetylation produced $\mathbf{2}$. All three compounds were prepared in peracetylated form in order to facilitate passive diffusion across cell membranes. ${ }^{3-5}$

Compounds 1-3 were initially screened for metabolic incorporation into Jurkat glycans by flow cytometry. Briefly, the cells were treated with 1-3 at concentrations of 10-200 $\mu \mathrm{M}$ for a period of up to 3 days, then reacted by Staudinger ligation with a phosphine reagent bearing the FLAG peptide. ${ }^{3-5}$ The cells were then labeled with a FITC-conjugated $\alpha$-FLAG antibody and analyzed by flow cytometry. Significant fluorescence was only observed after treatment with $\mathbf{3}$ at concentrations above $100 \mu \mathrm{M}$ (supporting information); compounds $\mathbf{1}$ and $\mathbf{2}$ did not appear to label cell surface glycans at any concentration tested. Interestingly, we determined that the observed signal resulted from cell death upon treatment with $\mathbf{3}$ at concentrations above $100 \mu \mathrm{M}$, as determined by propidium iodide staining (supporting information). By contrast, compounds $\mathbf{1}$ and $\mathbf{2}$ did not produce any adverse cellular effects at a concentration of $200 \mu \mathrm{M}$, suggesting that the toxicity of $\mathbf{3}$ is related to its cellular metabolism.

To identify the nature of the azide-labeled Jurkat glycoconjugates, we analyzed cell lysates by Western blot. Jurkat cells were cultured with $125 \mu \mathrm{M} \mathrm{1,2}$ or 3 for $72 \mathrm{~h}$ and then lysed with NP-40, a detergent known to be compatible with click chemistry ${ }^{15}$. The lysates were reacted with biotin-alkyne derivative $\mathbf{4}$ using the conditions for click chemistry suggested by Cravatt ${ }^{15}$ (Fig. 2), and the Western blot was probed with an $\alpha$-biotin antibody-horseradish 
peroxidase (HRP) conjugate. Significant glycoprotein labeling was only observed in lysates from cells treated with 6-azido fucose analog 3 (Fig. 3).

To provide direct evidence of the incorporation of $\mathbf{3}$ into Jurkat glycoproteins, lysates from cells cultured with 3 or the control, peracetylated fucose ( $\mathrm{Ac}_{4} \mathrm{Fuc}$ ) were subjected to mild acid hydrolys. The hydrolysates were analyzed by high $\mathrm{pH}$ anion exchange chromatography followed by pulsed amperometric detection (HPAEC-PAD). ${ }^{17}$ As shown in Fig. 4, hydrolysates from Jurkat cells treated with compound $\mathbf{3}$ contained 6-azido fucose (A) whereas hydrolysates from $\mathrm{Ac}_{4}$ Fuc-treated cells did not (B). Integration of the peaks corresponding to native fucose and 6-azido fucose in Fig. 4A suggested that the azido derivative replaced up to $40 \%$ of fucose residues under these conditions (supporting information).

In conclusion, fucosylated glycoproteins can be labeled in human cells with $\mathbf{3}$, whereas other isomers (compounds $\mathbf{1}$ and $\mathbf{2}$ ) are inactive. This must reflect the unnatural substrate tolerances of the salvage pathway enzymes, although the specific bottlenecks for the 2- and 4-azido analogs are not yet known. Furthermore, cell surface labeling with compound $\mathbf{3}$ likely reflects glycoprotein labeling, but a contribution from glycolipids is also possible and should be explored. Finally, the toxicity of compound $\mathbf{3}$ is a matter of interest and also concern. The correlation of metabolic conversion with toxicity suggests a specific mechanism involving either enzyme inhibition or altered protein function as a result of the azido sugar modification. These areas warrant further study in order to define the full potential of compound $\mathbf{3}$ for profiling protein fucosylation in living systems.

\section{Supplementary Material}

Refer to Web version on PubMed Central for supplementary material.

\section{Acknowledgments}

We thank Dr. Cheryl McVaugh for a critical reading of the manuscript and Nick Agard for compound $\mathbf{4}$ and the triazolyl ligand. This work was supported by a grant from the NIH to C.R.B. (GM66047). S.C.H. is supported by an NSF predoctoral fellowship.

\section{References}

1. Apweiler R, Hermjakob H, Sharon N. Biochim Biophys Acta. 1999; 1473:4-8. [PubMed: 10580125]

2. Prescher JA, Bertozzi CR. Nat Chem Biol. 2005; 1:13-21. [PubMed: 16407987]

3. Dube DH, Prescher JA, Quang CN, Bertozzi CR. Proc Natl Acad Sci USA. 2006; 103:4819-4824. [PubMed: 16549800]

4. Prescher JA, Dube DH, Bertozzi CR. Nature. 2004; 430:873-877. [PubMed: 15318217]

5. Vocadlo DJ, Hang HC, Kim EJ, Hanover JA, Bertozzi CR. Proc Natl Acad Sci USA. 2003; 100:9116-9121. [PubMed: 12874386]

6. Rosen SD. Annu Rev Immunol. 2004; 22:129-56. [PubMed: 15032576]

7. Kim YJ, Varki A. Glycoconj J. 1997; 5:569-576. [PubMed: 9298689]

8. Becker DJ, Lowe JB. Glycobiology. 2003; 13:41R-53R.

9. Everett JN, Kannagi R, Hakomori SI, Krantz MJ, Fuks A. J Immunol. 1985; 135:1911-1913. [PubMed: 2410505]

10. Fuster MM, Esko JD. Nat Rev Cancer. 2005; 5:526-542. [PubMed: 16069816]

11. Shi S, Stanley P. Proc Natl Acad Sci USA. 2003; 100:5234-5239. [PubMed: 12697902] 
12. a) Srivastava G, Kaur KJ, Hindsgaul O, Palcic MM. J Biol Chem. 1992; 267:22356-22361. [PubMed: 1429589] b) Vogel C, Bergemann C, Otter A-J, Lindhorst TK, Thiem J, Dahlhoff WV, Hällgren C, Palcic MM, Hindsgaul O. Liebigs Ann. 1997:601-612.

13. Anisuzzaman AKM, Horton D. Carbohydr Res. 1987; 169:258-262.

14. Komba S, Ishida H, Kiso M, Hasegawa A. Bioorg Med Chem. 1996; 4:1833-1847. [PubMed: 9007268]

15. Speers AE, Cravatt BF. Chem Biol. 2004; 11:535-546. [PubMed: 15123248]

16. Chan TR, Hilgraf R, Sharpless KB, Fokin VV. Org Lett. 2004; 6:2853-2855. [PubMed: 15330631]

17. Cataldi TR, Campa C, De Benedetto GE. J Anal Chem. 2000; 368:739-758. 


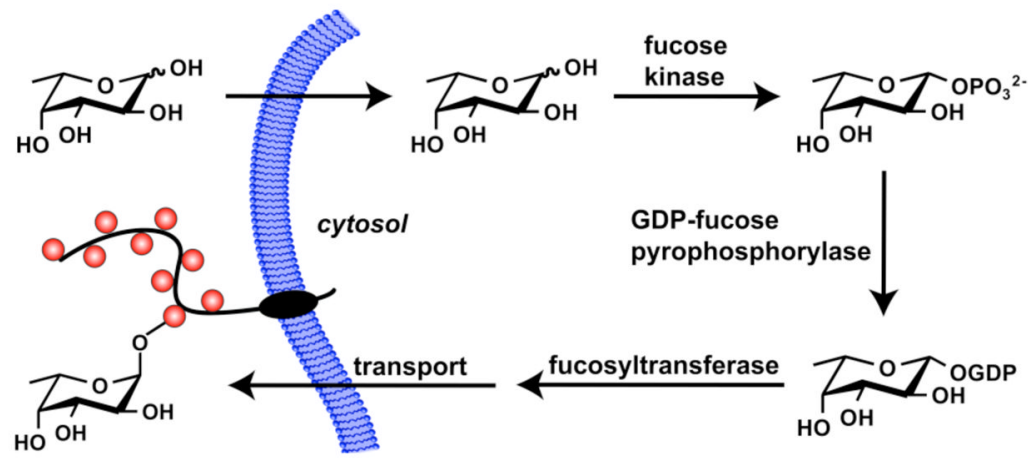

Figure 1.

Fucose salvage pathway. 


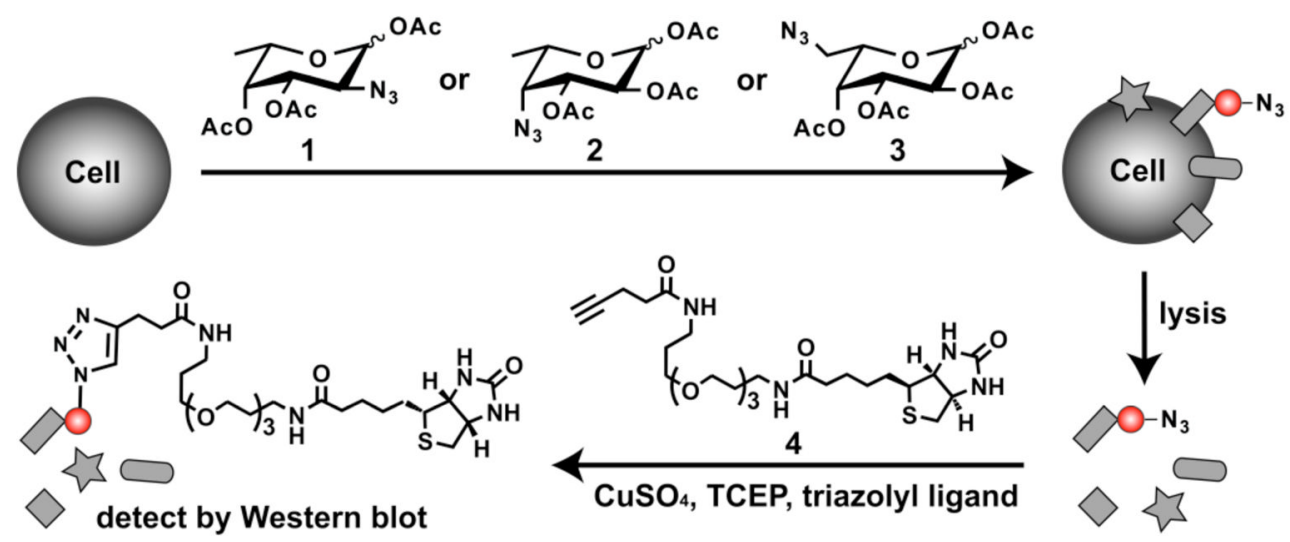

Figure 2.

Metabolic labeling of cellular glycoproteins with azido fucose analogs. Jurkat cells were cultured with azido fucose derivatives, 1,2 , or 3 , and their lysates were reacted with $\mathbf{4}$ in the presence of $\mathrm{CuSO}_{4}$, TCEP, and triazolyl ligand. ${ }^{16}$ The labeled lysates were analyzed by Western blot with an $\alpha$-biotin antibody-HRP conjugate. 


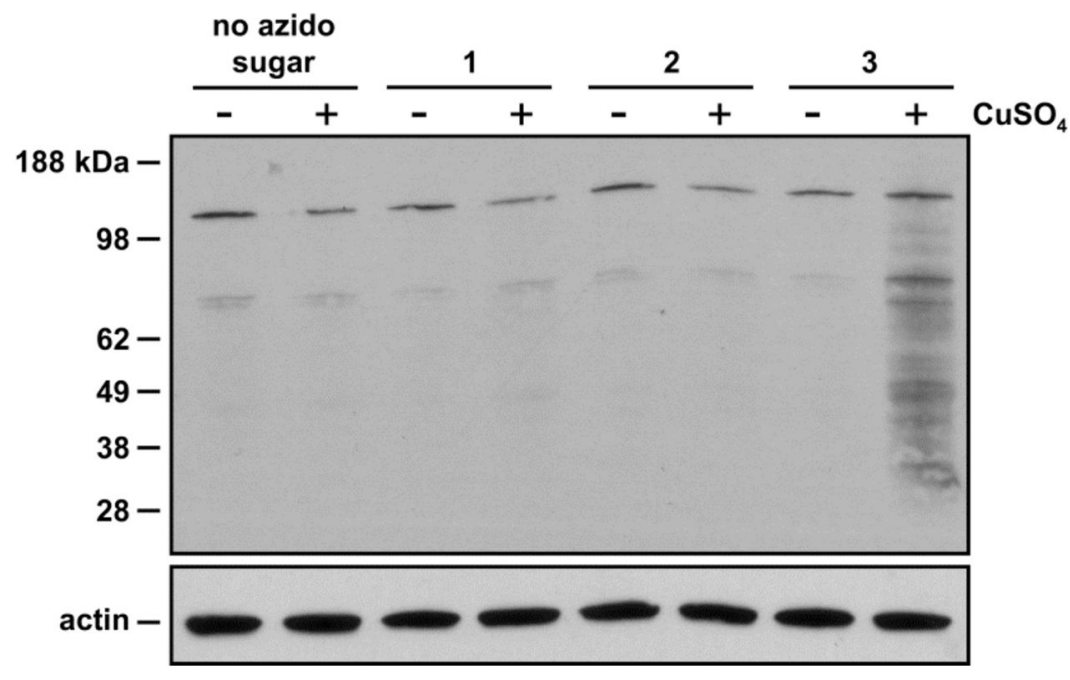

Figure 3.

Western blot analysis of lysates from Jurkat cells treated with 1, 2, 3 or buffer (no azido sugar). Jurkat lysates were reacted with a biotin-alkyne probe in the presence (+) or absence $(-)$ of $\mathrm{CuSO}_{4}$. The blot was probed with an $\alpha$-biotin antibody conjugated to horseradish peroxidase (HRP) (upper panel), then stripped and re-probed with an $\alpha$-actin antibody as a protein loading control (lower panel). 


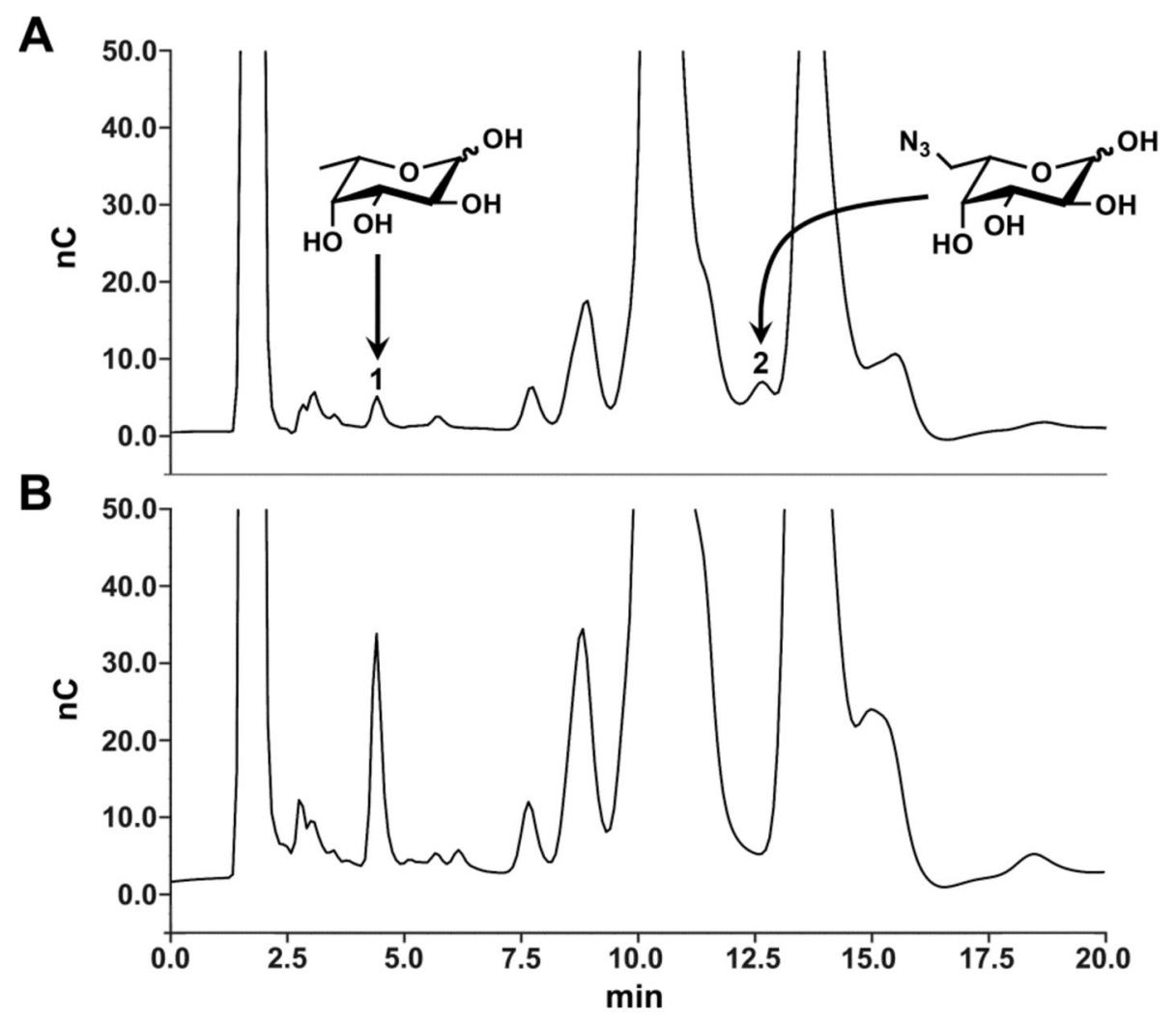

Figure 4.

HPAEC-PAD analysis of hydrolysates from Jurkat cells incubated with (A) compound $\mathbf{3}$ or (B) $\mathrm{Ac}_{4}$ Fuc. The peaks labeled 1 and 2 correspond to native fucose and 6-azido fucose, respectively, as determined by analysis of authentic standards. Details are in the supporting information. 


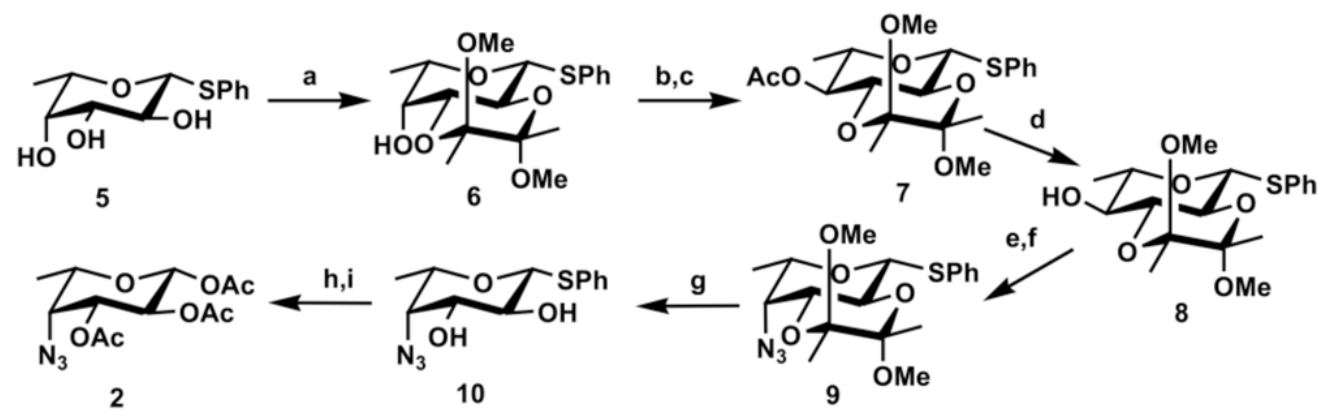

Scheme 1.

Reagents: a) butane-2,3-dione, trimethylorthoformate, $\mathrm{CSA}, \mathrm{MeOH}, 87 \%$. b) $\mathrm{Tf}_{2} \mathrm{O}$, pyr. c) toluene, $\mathrm{Bu}_{4} \mathrm{NOAc}, 64 \%$ (2 steps). d) $\mathrm{MeOH}, \mathrm{NaOMe}, 87 \%$. e) $\mathrm{Tf}_{2} \mathrm{O}$, pyridine. f) $\mathrm{LiN}_{3}$, DMF, $88 \%$ (2 steps). g) TFA, $\mathrm{H}_{2} \mathrm{O}, 98 \%$. h) NBS, acetone, $\mathrm{H}_{2} \mathrm{O}$. i) $\mathrm{Ac}_{2} \mathrm{O}$, pyridine, $58 \%$ (2 steps). 